Dermatologische Zeitschrift. 1919;28:I-IV

\title{
Contents, Vol. 28, 1919
}

\section{Dermatologische Zeitschrift} Begründet von O. LASSAR

Unter Mitwirkung von A. Alexander-Berlin; J. Almkvist-Stockholm; Ed. Arning-Hamburg; F. Bering-Essen; S. Bettmann-Heidelberg; B. Bloch- Zurich; C. Bruhns-Berlin; A. Buschke-Berlin; C. Cronquist-Malmö; J. Darier-Paris; K. Dohi-Tokio; S. Ehrmann-Wien; J. Fabry-Dortmund; W. Frieboes-Rostock; W. Gennerich-Kiel; C. Grouven-Haüe; L. Hauck-Erlangen; J. Heller-Berlin; W. Heuck-München; H- Hübner-Elberfeld; A. Jesionek-Gießen; F. Juliusberg-Posen; F. KyrleWien; P. Linser-Tiibingen; F. Lewandowsky-Basel; R. Matzenauer-Graz; H. Meyer-Kis/81; E. Meirowsky-Köln a. Rh.; P. Mul-zer-München; G. Nobl-Wien; A. Pasini-Mailand; F. Pin 1/8usBerlin; R. Polland-Graz; C. Rasch-Kopenhageií; E. Riecke-Göttin en; G. Rost-Freiburg; A. Ruete-Marburg; J. Schaeffer-Breslau; G. Scherber-Wien; W. I. Schmidt-Bonn; W. ScholtzKöuigsberg i. Pr.; B. Spiethoff-Jena; C. Stern-Düsseldorf; E. Tomasczewski-Berlin; L. TòrökBudapest; H. Vörner-L·eipzig; J. Werther-Dresden; Ch. J. White-Boston; K. Zieler-Wür ¿burg ; F. Zinsser-Köln a. R. und in Gemeinschaft mit

G. Amdt A. Blaschko S. Ehrmann K. Herxheimer V. Klingmüllei

Berlin Berlin Wien Frankfurt a. M. Kiel

Herausgegeben von

Erich Hoffmann

Bonn

Bd. XXVIII.

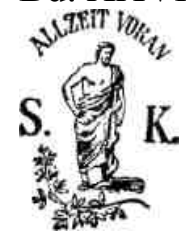

BERLIN 1919

VERLAG VON S. KARGER

KARLSTKASStí lõ

Mit zahlreichen Abbildvngen im Text und 6 ïafeln.

Inhalts -Verzeichnis.

Originalarbeiten. $\quad \wedge \mathbf{i}^{1 / 8}$

A r z t, L. und Fuhs, H., Über einen Mikrosporieherd in

Wien .319

Boas, H., Ein Fall von Dermatitis herpetiformis mit

Psychose $\quad 325$

ïrieboes, W., Beiträge zur Anatomie und Biologie der

; Haut. I. Das Rongalitweißbild der Hautnerven. (Hierzu

Taf. 111-VI) .267 
Gartner, W., Was lehrt die serologische Sonderstellung

des Liquor cerebrospinalis und des Kammerwassers bei

Typhus, Fleckfieber und Syphilis für die Behandlimg der

Syphilis? $\quad 147$

H e c h t , H., Ein Beitrag zur Reinfectio syphilitica . . . . 193

$\mathrm{H}$ e 11 e , H., Über einen Fall von primärer Sarkomatosis cutis

multiplex 197

Heller, J., Die Stellung des Facharztes bei der Begut-aehtung der Nichtinfektiosität eines

Patienten zu dem Paragraphen 3 (Gefährdungsparagraphen) des neuen Gesetzes zur Bekämpfung der Geschlechtskrankheiten 65

Hoffmann, Erich, Über eine nach innen gerichtete biolo-

gische Schutzfunktion der Haut (Esophylaxie) nebst Be-

merkungen über die Entstehung der Paralyse 257

-, -, Über Hautschädigungen durch Kalkstickstoffdünger 38

H o f m a n n , Edmund, Über einen Todesfall nach Silber-

salvarsan 281

Husen,J. van, Ein Beitrag zur Kenntnis des Boeckschen

MiHarlupoids und seiner Beziehung zu Erkrankungen

anderer Organe. (Hierzu Taf. I) 1

Kaufmann, R., Über Versuche, Neosalvarsan- und Salvar-

sanlösungen haltbarer zu machen 35

Ledermann, R., Über dermato-therapeutische Ersatz -

präparate $\quad 96$

Lutz,W., Paroxysmale Hämoglobinurie und Syphilis . 136

Allo Rechte vorbekalten.

Ge $<$ iiuckt bei Imberg \& Leíson G. m. b. H. in Berlin SW. 48.

- IV -

Séite

Moos und Warstat. Die Behandlung der Syphilis

des Zentralnervensystems mit epiduralen Salvarsan-

injektionen ...291

Muschter, Ein Beitrag zu dem Krankheitsbild des Lupus

pernio. (Hierzu Taf. II.) 203

P i c h 1 e r , K., Berufsschwiele auf der Scheitelhöhe durch

Lastentragen, besonders bei Schweinemägden $\quad 73$

Pinkus, F., Die Pohl-Pinkussche Marke an den Haaren

beim akuten Haarausfall nach Grippe 106

P o 11 a n d, R., Eine entzündliche Lymphgefäßgeschwulst am

Oberschenkel 31

--, -, Neue klinische Beiträge zur Klarstellung des Unterschiedes zwischen Dermatosis dysmenorrhoica, Herpes neuroticus und Selbstbeschädigung 77

R u e t e, Zur Frage der Terpentinbehandlung 28

Sluczewski, A., Zur Thorium-X-Doramad-Behandlung

bei Dermatosen 211 
Stutzin, J. J., Über einige urologische Grenzfragen . . 340 Zurhelle,E., Zur klinischen Bewertung der Ausflockungs-

reaktion auf Syphilis nach Sachs und Georgi .... 129

Literarische Notiz 39

H. E. Schmidt $\dagger \quad 128$

Gesellsehaf tsberichte.

Verhandlungen der Berliner dermatologischen Gesellschaft.

Sitzung vom 2. II. $1919 \quad 230$

Sitzung vom 8. IV. $1919 \quad 354$

Dänische dermatologische Gesellschaft. Sitzungen vom Ok-

tober und November 1916, 18. III. 1919, 8. IV. 1919237

Periodische Literatur 45, 116, 186, 253, 309, 359

Buchanzeigen 61, 369

Tagesnachriehten $\quad 64,128, \quad 318$

Personalien 186, 318, 372

Namen- und Sachregister 